\title{
Direct Immunofluorescence Studies in Lichen Planus
}

\author{
Supriya JAIN ${ }^{1}$ (D), Vijaya BASAVARAJ ${ }^{2}$ \\ Department of Pathology, 'Bharati Vidyapeeth (deemed to be university) Medical College and Hospital, SANGLI, INDIA, \\ 2J.S.S Medical College, J.S.S. University, MYSORE, INDIA
}

\begin{abstract}
Objective: Lichen planus is a common, usually intensely pruritic, symmetrical, papulosquamous dermatosis. Direct immunofluorescence studies in patients with lichen planus shows deposition of multiple immunoglobulins and fibrin at the dermoepidermal junction and in the colloid bodies.

Material and Method: Histopathological features were analysed in 100 cases of lichen planus which were sent for routine histology. Direct immunofluorescence studies were done in 22 out of the 100 cases and the features were analysed. Clinical data was recorded from patient files.

Results: Positive direct immunofluorescence was seen in $78.5 \%$ of the cases. Deposits at the dermoepidermal junction and colloid bodies were detected in $88 \%$ and $40 \%$ of the cases respectively. IgG, IgM and C3 deposition was seen in $88 \%, 70 \%$ and $24 \%$ respectively. IgA was negative in all the cases.

Conclusion: The linear and shaggy deposition of immunoreactants in a discontinuous form along the dermoepidermal junction and in the colloid bodies were indicators in support of lichen planus along with the characteristic histopathological findings. In lupus erythematosus, linear and granular deposition of immunoglobulins in a continuous form is found along the dermoepidermal junction. Direct immunofluorescence studies are of immense help in disease differentiation in cases of interface dermatitis with no specific histological or clinical characteristics and in cases with ambiguous features.
\end{abstract}

Key Words: Lichen planus, Lupus erythematosus, Direct immunofluorescence

\section{INTRODUCTION}

Lichen Planus (LP) is a common inflammatory disease of unknown etiology characterized by violaceous and flattopped papules, which are usually pruritic. A network of fine white lines (Wickham's striae) may be seen on the surface of the papules. There is a predilection for the flexor surface of the wrists, the trunk, the thighs and the genitalia (1). LP frequently occurs between 30 and 60 years of age. The diagnosis of LP can routinely be done based on clinical and histopathological examination. Many centers have routinely employed direct immunofluorescence (DIF) studies in cases of LP. DIF studies are essentially required in cases where specific clinical or histological features are not present. Cases of interface dermatitis can be very intriguing with ambiguous features in other diseases. e.g. lupus erythematosus (LE). DIF in previous studies have shown immunoglobulins, complement and fibrin deposition in the colloid bodies (CB) and at the dermoepidermal junction (DEJ) (2). This study was undertaken to evaluate the utility of DIF studies in LP, and whether DIF studies are really required in LP.

(Turk Patoloji Derg 2019, 35:193-197)

Received : 21.09.2018 Accepted : 06.03.2019

\section{MATERIAL and METHODS}

The study was undertaken at the department of pathology. Clinically diagnosed cases of LP and its variants which were sent for histopathological study and DIF studies were included in the study. The clinical findings and histopathologic features of a total of 100 cases were studied. DIF studies were performed on 22 cases and the data for DIF was analyzed in 22 cases.

The skin punch biopsy specimen for histopathological study was received in $10 \%$ formalin and processed for routine histology after $24 \mathrm{hrs}$ of fixation in formalin and studied by light microscopy. The skin punch biopsy specimen was obtained in normal saline for DIF studies. The specimen for DIF studies was washed thrice in Phosphate Buffer Saline (PBS) and snap frozen in OCT (Optimum Cutting Temperature) medium followed by cutting of 4 micron thick sections on 4 slides labeled IgG, IgA, IgM and C3. This was again washed in PBS and treated with FITC (Fluorescein Iso Thio Cyanate) labelled antisera respectively and incubated for $1 \mathrm{hr}$ at 37 degrees followed by washing thrice with PBS and then evaluated under a fluorescent microscope.

Correspondence: Supriya JAIN

Department of Pathology, Bharati Vidyapeeth (deemed to be university)

Medical College and Hospital, SANGLI, INDIA

E-mail: sups.ain@gmail.com Phone: +99 86999968 


\section{RESULTS}

Out of the 100 cases (52 males and 48 females) of LP, DIF studies were done in 22 cases (10 males and 12 females). Their age ranged from 13 years to 80 years, and the duration of the lesion ranged from 15 days to 1 year. The most common site of involvement was the extremities (67 cases) followed by the trunk (43 cases). Oral lesions were seen in 20 cases and genital involvement in 8 cases, as shown in Table I. Itching was the most common clinical complaint seen in 88 cases followed by pigmentation in 42 cases. 50 cases showed papular lesions followed by hyperpigmented patches in 42 cases, as shown in Table II.

Biopsies obtained from the lesions showed various histopathological features. The epidermis showed compact orthokeratosis, wedge-shaped hypergranulosis, mild acanthosis and basal cell vacuolar degeneration in 72 cases. The remaining cases had only some of these features. Pointed rete ridges were seen in 41 cases. The dermis showed a band-like lymphohistiocytic infiltrate along the dermoepidermal junction (DEJ) abutting the basal cells and sometimes obscuring the $\mathrm{DEJ}$ in 70 cases. CB were seen in 42 cases. Melanin pigment incontinence was seen in 74

Table I: The sites of involvement $(n=100)$.

\begin{tabular}{lc}
\hline Site of involvement & $\begin{array}{c}\text { Percentage of cases } \\
\text { (No. of cases) }\end{array}$ \\
\hline Extremities & $67 \%(67)$ \\
\hline Trunk & $43 \%(43)$ \\
\hline Oral lesions & $20 \%(20)$ \\
\hline Genital involvement & $8 \%(8)$ \\
\hline
\end{tabular}

cases, as shown in Table III. The histological features were as shown in Figure 1. A few cases had features like occasional cells showing basal cell vacuolar degeneration or mild pigment incontinence with a few scattered lymphocytes in the upper dermis and not meeting the criteria for the diagnosis of classical lichen planus. These were the cases which required DIF studies to arrive at a final diagnosis.

Out of the 22 cases sent for DIF studies, 17 cases showed deposition of immunoglobulins accounting to positive DIF studies in $77 \%$ of the cases. The pattern of deposition of immunoglobulins was focal and shaggy along the DEJ with irregular fine extensions into the papillary dermis. Of the 17 cases positive for DIF, deposition of IgG was seen in 15 cases accounting for $88 \%$, deposition of IgM was seen in 12 cases accounting for $70 \%$, and deposition of $\mathrm{C} 3$ was seen in 4 cases accounting for $24 \%$ of the cases. Seven cases showed deposition of immunoreactants in the colloid bodies accounting to $40 \%$ of the cases. Of the immunoreactants which were deposited in the colloid bodies, IgG was seen in all 7 cases, IgM in 4 cases and $\mathrm{C} 3$ in 4 cases. (Figure 2-4) IgA was negative in all the cases. The distribution of immunoreactants was as shown in Table IV.

Table II: The clinical presentation of various lesions $(n=100)$.

\begin{tabular}{lc}
\hline Clinical presentation & $\begin{array}{c}\text { Percentage of cases } \\
\text { (No. of cases) }\end{array}$ \\
\hline Itching & $88 \%(88)$ \\
\hline Itching with pigmentation of skin & $42 \%(42)$ \\
\hline Papular lesions & $50 \%(50)$ \\
\hline Hyperpigmented patches & $42 \%(42)$ \\
\hline
\end{tabular}

Table III: The histopathological features $(\mathrm{n}=100)$.

\begin{tabular}{lc}
\hline Histopathological feature & Percentage of cases (No. of cases) \\
\hline Orthokeratosis, hypergranulosis, acanthosis and basal cell vacuolar degeneration & $72 \%(72)$ \\
\hline Melanin pigment incontinence & $74 \%(74)$ \\
\hline Pointed rete ridges & $41 \%(41)$ \\
\hline Colloid bodies & $42 \%(42)$ \\
\hline Band of lympho-histiocytic infiltrate along the upper dermis abutting basal cells & $70 \%(70)$ \\
\hline
\end{tabular}

Table IV: The distribution of various immunoglobulins $(n=17 / 22)$.

\begin{tabular}{lcccc}
\hline & IgG & IgM & C3 & IgA \\
\hline DEJ & $88 \%(15 / 17)$ & $70 \%(12 / 17)$ & $24 \%(4 / 17)$ & 0 \\
\hline Colloid Bodies & $41 \%(7 / 17)$ & $24 \%(4 / 17)$ & $24 \%(4 / 17)$ & 0 \\
\hline
\end{tabular}

DEJ: Dermoepidermal junction. 


\section{DISCUSSION}

LP is an immune mediated disease which on DIF studies shows a linear and shaggy broad band of staining with fibrinogen, immunoglobulins and complement along the DEJ. CB in the papillary dermis may show deposition of immunoglobulins. $\mathrm{CB}$, also known as Civatte bodies, are eosinophilic hyaline ovoid bodies which are often found in the subepidermal papillary regions or sometimes in the epidermis. They are usually seen in LP and LE (3). They can also be found in several dermatoses such as erythema multiforme (EM), bullous pemphigoid (BP) and diseases with suprabasal clefts (4). CB are generally believed to be derived from two origins. The first type originates from

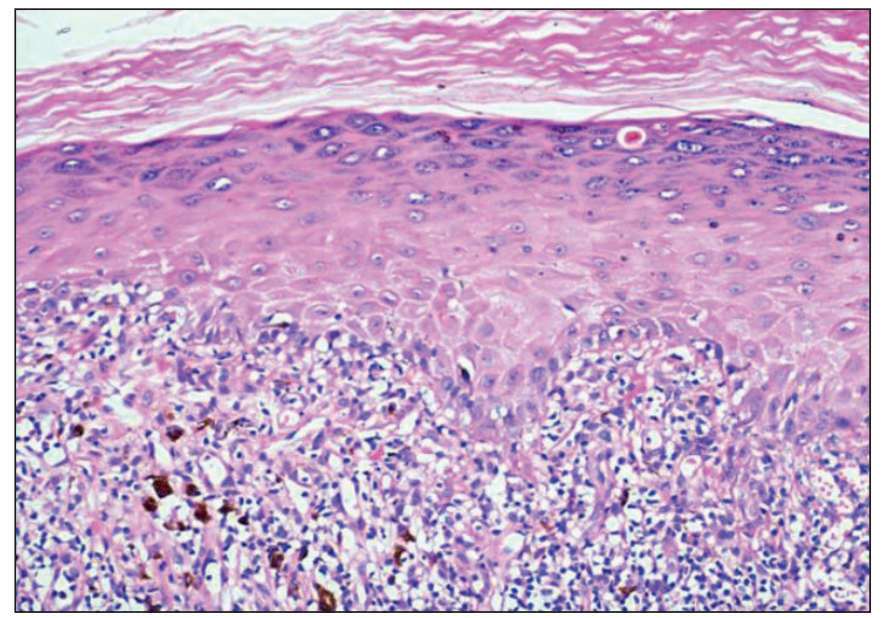

Figure 1: High power view showing epidermal acanthosis and hyperkeratosis along with saw toothing of rete ridges. There is basal cell vacuolar degeneration with a band of lympho-histiocytic infiltrate at the dermoepidermal junction. Melanin pigment incontinence and Civatte bodies are also noted (H\&E; x200).

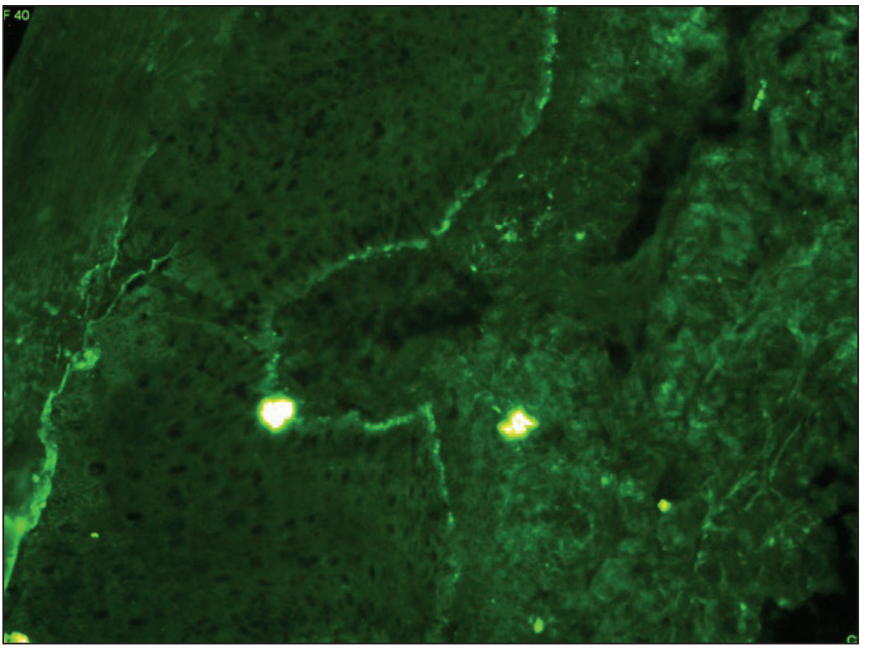

Figure 3: Deposition of C3 in a continuous linear and granular pattern along the dermoepidermal junction (DIF; x200). apoptosis of keratinocytes causes by epithelium damage created by circulating antibodies (5). CB of this type are usually found locally both in the epidermis and papillary dermis. The other origin derives from the destruction of thickened basement membranes which are found only in the papillary dermis. DIF studies help in differentiating these conditions and hence is essential to arrive at a final diagnosis in cases with overlapping features.

In our study, the positive DIF yield was $78.5 \%$ whereas in many previous studies the positive yield ranged from around $37 \%$ to $97 \%(2,6)$. In most of the previous studies the specimen was mostly from oral mucosal lesions.

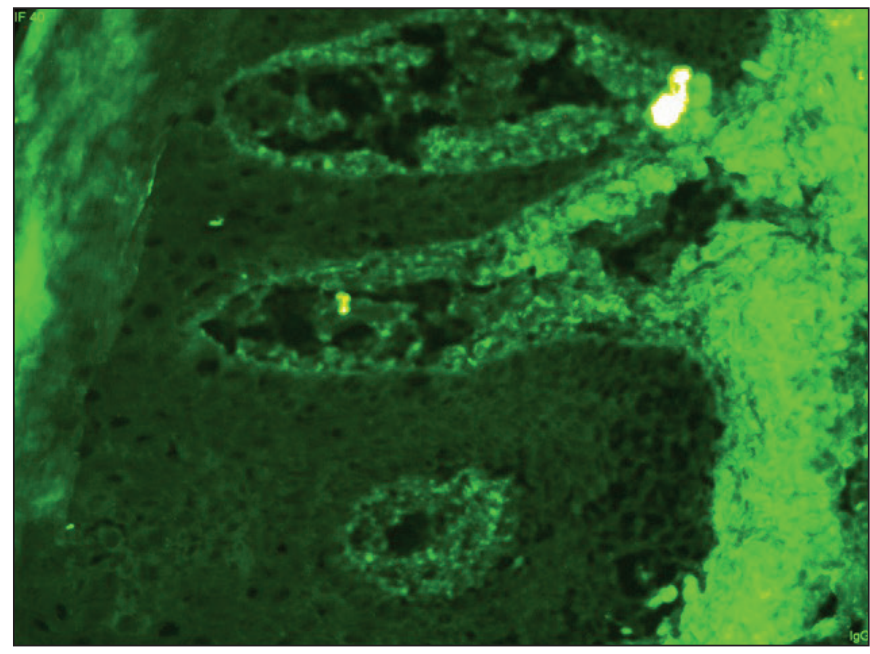

Figure 2: Deposition of IgG in a linear, granular and discontinuous pattern along the dermoepidermal junction with extension into papillary dermis in LP (DIF, x200).

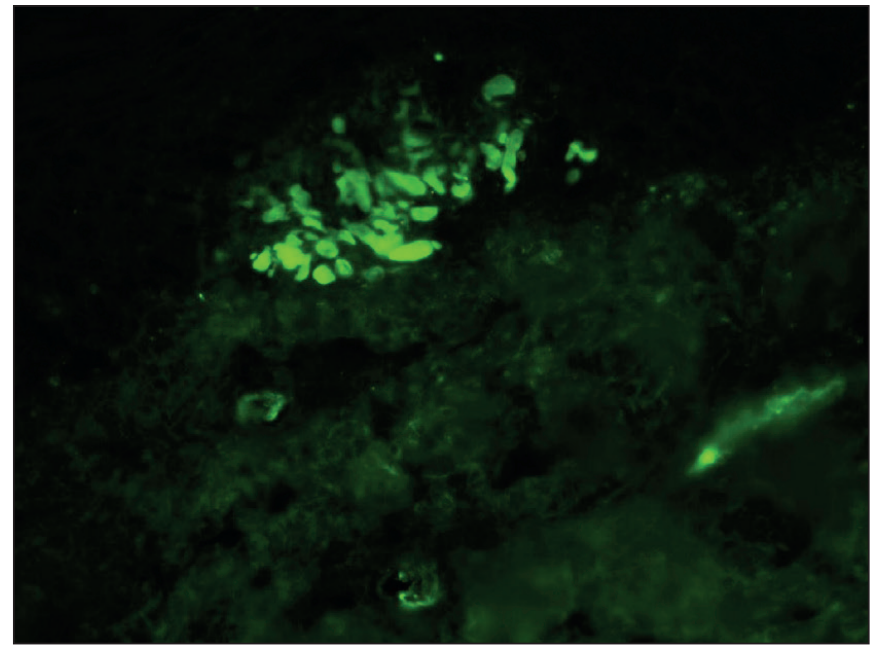

Figure 4: Deposition of IgM in the colloid bodies (DIF; x200). 
Annesi et al. reported a positive yield of DIF in $71 \%$ of the cases from scalp lesions with scarring alopecia due to LP and Kulthanan et al. have reported a positive yield in $73 \%$ of the cases from glabrous skin $(2,6)$. Previous DIF studies have resulted in deposition of immunoglobulins in around $90 \%$ of the cases which is similar to study (2).

Our study showed deposition of immunoreactants and complement at DEJ and in the CB. The most common immunoreactant was IgG, which was positive in $88 \%$ of the cases. The other immunoreactants in decreasing order of frequency were IgM and C3. IgA was negative in all cases. The results are similar to the results in most of the previous studies where IgG was the most common immunoglobulin deposit along DEJ after fibrinogen. Previous studies have not included uniform reports of the class of immunoreactant deposited in CB but in our study it was IgG.

Helanders and Rogers have suggested that the diagnostic sensitivity of LP by presence of $\mathrm{CB}$ alone is poorer, and it is better defined by shaggy deposits of immunoglobulins and complement along DEJ. However, CB in LP demonstrate a tendency to cluster in groups of 10 or more in the papillary dermis (7).

Morphologically identical deposits of immunoglobulins in colloid bodies and at the DEJ may be found in LP and LE, and DIF studies play a significant role in distinguishing them. This distinction becomes all the more important in cases where histopathological and clinical findings are overlapping and non diagnostic.

DIF studies in LP show deposition of immunoreactants and fibrin along the DEJ in a broad discontinuous and shaggy pattern along with colloid body deposition. LE lesions are likely to contain linear band-like deposits of Ig and C3 in the DEJ. If IgM is present, there is a high probability that the patient has LE, and C3 deposition also occurs more frequently in LE than in LP. The pattern of staining in LE is linear, granular, broad, discontinuous band-like but it may also be smooth and continuous in occasional cases.

Schiodt et al. have recommended that granular staining along DEJ with at least one immunoglobulin in addition to C3 is necessary for diagnosis of LE (8).

Kok et al. evaluated both direct and indirect IF studies on patients with cutaneous and oral lichen planus and found that although DIF studies showed deposition of fibrin in a granular or shaggy pattern in a considerable percentage of cases, other immunoglobulins were seen in only a minimal number of cases. They did not find a significant level of circulating immune complexes in patients with LP. These observations made them rule out the possibility of immune complexes playing a significant role in the pathogenesis of LP. The authors comment that deposition of fibrinogen is nonspecific and can also be seen in the skin and corneal wound and in many immune mediated reactions and hence they consider that fibrin deposition is non-specific and a part of the homeostatic mechanism of the body (9).

Immunoreactant deposits at $\mathrm{CB}$ alone can be found in various diseases but a strong intensity and high quantity favor the diagnosis of interface dermatitis. CB plus DEJ deposits are more common in interface dermatitis than in any other disease. Between lichen planus and lupus erythematosus, $\mathrm{CB}$ alone is more common in $\mathrm{LP}$ whereas the combination of $\mathrm{CB}$ plus DEJ and superficial blood vessels is more common in LE. The most common pattern in both diseases is CB plus DEJ. Although both LP and LE show overlapping results regarding the type of immunoglobulin deposition on DIF, the pattern of deposition makes the difference. In our study, LP consistently showed a shaggy deposition along the DEJ with irregular extensions into papillary dermis as shown in Figure 2 and 3. The quantity and intensity of CB in LP is higher than in LE and is shown in Figure 4. In comparison, cases of LE show broad, continuous, linear and granular deposition of immunoreactants confined to the basement membrane zone without extensions into the papillary dermis or epidermis as shown in Figure 5. The shaggy irregular deposits are characteristic of LP. LE lesions are likely to contain deposits of immunoglobulins and $\mathrm{C} 3$ in the DEJ. If IgM is present in a discontinuous band-like pattern, there is high probability that the patient has LE. C3 deposition occurs more frequently in LE than in LP

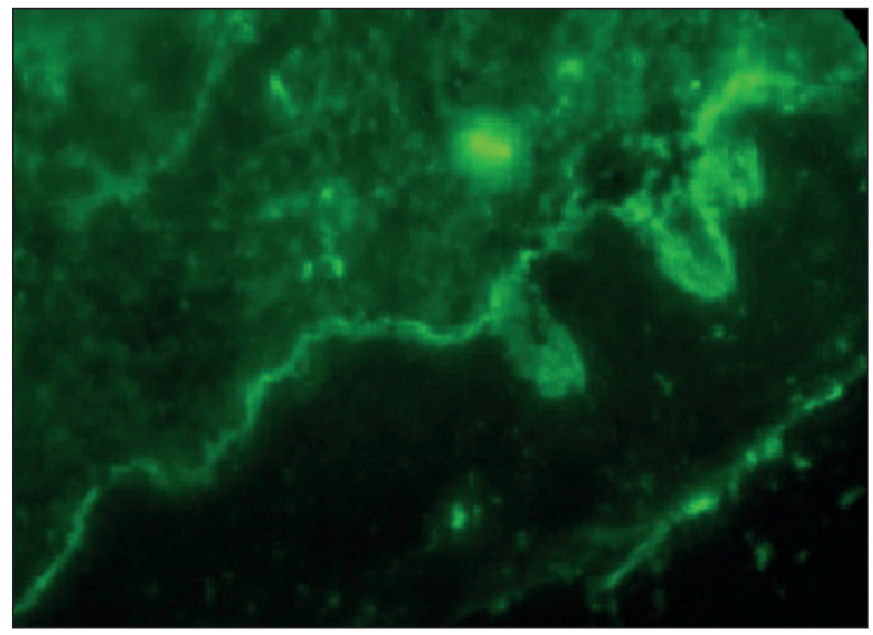

Figure 5: Deposition of IgG in a broad, continuous, linear and granular pattern along the dermoepidermal junction in a case of lupus erythematosus (DIF; x400). 
Oral LP poses a diagnostic dilemma clinically. The clinical differential diagnosis of oral lichen planus includes lichenoid drug eruptions, lichenoid lesions associated with contact hypersensitivity to restorative materials, leukoplakia, lupus erythematosus and graft versus host disease (10). DIF studies help in distinguishing oral LP from other lesions such as pempgius vulgaris, benign mucous membrane pemphigoid and linear IgA bullous dermatosis (11). DIF has a more significant role in the diagnosis of oral LP than cutaneous LP.

In summary, the positive yield of direct immunofluorescence in the present study was $78.5 \%$. Shaggy deposition of immunoreactants and especially $\operatorname{IgG}$ and the deposition of immunoreactants in colloid bodies were the indicators of diagnosis of LP with the support of characteristic histopathology findings. In the present study, histopathological features were diagnostic in the majority of the cases. DIF studies were supplementary in such cases. However, DIF studies are of immense help in cases with ambiguous histological and clinical features. Hence, the authors suggest that DIF studies need not be routinely employed in all cases of LP as they are not cost-effective and should be resorted to only when the histopathological study and clinical features are non-contributory.

We would like to conclude that DIF studies in cases of interface dermatitis are required only in cases where the histopathology is ambiguous. DIF studies are more beneficial in oral LP as compared to cutaneous LP and DIF studies are of immense help in cases with overlapping features between LP and LE. The limitation of the present study was the non availability of anti fibrinogen. However, the pattern of deposition of other immunoglobulins in a shaggy manner was conclusive enough to support the diagnosis of LP.

\section{CONFLICTS of INTEREST}

The authors declare no conflict of interest

\section{REFERENCES}

1. Weedon D. Weedon's skin pathology essentials. 3rd ed. London: Churchill Livingstone Elsevier. 2010

2. Kulthanan K, Jiamton S, Varothai S, Pinkaew S, Sutthipinitharm P. Direct immunoflorescence studies in patients with lichen planus. Int J Dermatol. 2007;46:1237-41.

3. Horiguchi Y, Danno K, Ikai K, Imamura S. Colloid body formation in bullous pemphigoid. Arch Dermatol Res. 1985;277:167-73.

4. Sumegi I. Colloid bodies in dermatoses other than lichen planus. Acta Derm Venereol. 1982;62:125-31.

5. Leena C, Papapit T, Daranporn T, Pinkaew S, Kulthanan K. Diagnostic significance of colloid body deposition in direct Immunofluorescence. 2010;76:373-7.

6. Annessi G, Lombardo G, Gobello T, Puddu p. A clinicopathologic study of scarring alopecia due to lichen planus:Comparison with scarring alopecia in DLE and pseudopalade. Am J Dermatopathol. 1999;21:324-31.

7. Helander SD, Rogers RS. The sensitivity and specificity of direct immunofluorescence testing in disorders of mucous membranes. J Am Acad Dermatol. 1994;30:65-75.

8. Schiodt M, Holmstrup P, Dabelsteen E, Ullman S. Deposits of immunoglobulins, complement, and fibrinogen in oral lupus erythematosus, lichen planus, and leukoplakia. Oral Surg Oral Med Oral Pathol. 1981;51:603-8.

9. Kok SH, Wu YC, Chiu HC, Sun A, Lu YC. Immunopathological findings and immune complex in lichen planus. Derm Sinica.1987;5:1-8

10. Kumar S, Hiremath S, Kale AD, Hallikerimath S. Clinicopathological study to evaluate oral lichen planus for the establishment of clinical and histopathological diagnostic criteria. Turk Patoloji Derg. 2016;32:91-8.

11. Yamanaka Y, Yamashita M, Innocentini LMA, Macedo LD, Chahud F, Ribeiro-Silva A, Roselino AM, Rocha MJA, Motta AC. Direct Immunofluorescence as a Helpful Tool for the differential diagnosis of Oral Lichen Planus and Oral Lichenoid Lesions. Am J Dermatopathol. 2018;40:491-7. 